\title{
BMJ Open Feasibility, qualitative findings and satisfaction of a brief Tai Chi mind-body programme for veterans with post-traumatic stress symptoms
}

\author{
Barbara L Niles, ${ }^{1}$ DeAnna L Mori, ${ }^{2}$ Craig P Polizzi, ${ }^{3}$ Anica Pless Kaiser, ${ }^{1}$ \\ Annie M Ledoux, ${ }^{3}$ Chenchen Wang ${ }^{4}$
}

To cite: Niles BL, Mori DL, Polizzi CP, et al. Feasibility, qualitative findings and satisfaction of a brief Tai Chi mind-body programme for veterans with post-traumatic stress symptoms. BMJ Open 2016;6:e012464.

doi:10.1136/bmjopen-2016012464

- Prepublication history for this paper is available online. To view these files please visit the journal online (http://dx.doi.org/10.1136/ bmjopen-2016-012464).

Received 29 April 2016 Revised 10 August 2016 Accepted 4 October 2016

CrossMark

For numbered affiliations see end of article.

Correspondence to Dr Barbara L Niles; Barbara.Niles@va.gov

\section{ABSTRACT}

Objective: To examine feasibility, qualitative feedback and satisfaction associated with a 4-session

introduction to Tai Chi for veterans with post-traumatic stress symptoms.

Design: We observed and reported recruitment and retention rates, participant characteristics, adherence, and satisfaction across 2 cohorts. We also examined qualitative feedback provided by questionnaires, focus groups and individual interviews.

Main outcome measures: Rates of recruitment and retention, focus group and individual feedback interviews, self-reported satisfaction.

Participants: 17 veterans with post-traumatic stress symptoms.

Results: Almost 90\% (17/19) of those eligible following the telephone screen enrolled in the programme. Three-quarters $(76.4 \%)$ of the participants attended at least 3 of the 4 Tai Chi sessions. Qualitative data analysis revealed themes indicating favourable impressions of the Tai Chi sessions. In addition, participants reported feeling very engaged during the sessions, and found Tai Chi to be helpful for managing distressing symptoms (ie, intrusive thoughts, concentration difficulties, physiological arousal). Participants also reported high satisfaction: $93.8 \%$ endorsed being very or mostly satisfied with the programme. All participants $(100 \%)$ indicated that they would like to participate in future Tai Chi programmes and would recommend it to a friend.

Conclusions: Tai Chi appears to be feasible and safe for veterans with symptoms of post-traumatic stress disorder (PTSD), is perceived to be beneficial and is associated with high rates of satisfaction. This study highlights the need for future investigation of Tai Chi as a novel intervention to address symptoms of PTSD.

\section{INTRODUCTION}

The lifetime risk of developing posttraumatic stress disorder (PTSD) in the US general population is estimated to be $8.7 \% .^{1}$ In veterans, with higher exposure to
Strengths and limitations of this study

- This is the first study to examine feasibility, qualitative feedback and satisfaction associated with Tai Chi for veterans with post-traumatic stress symptoms. Tai Chi, an ancient Chinese form of exercise, may provide an appealing alternative to current empirically supported treatments for post-traumatic stress disorder (PTSD).

- Qualitative findings indicated that veterans perceived Tai Chi to help reduce several symptoms (ie, intrusive thoughts, concentration difficulties, physiological arousal). Satisfaction ratings were very high.

- The small sample and short four-session programme did not allow conclusions to be drawn regarding feasibility and efficacy of a typical, longer length Tai Chi intervention.

- This study provides preliminary indications that veterans with PTSD symptoms are interested in Tai Chi and suggests possible mechanisms of change (eg, reducing physiological arousal, improving comorbid conditions, increasing positive associations with warrior identity) to be examined in future rigorous trials.

traumatic events, the risk is higher, with an estimate of $23.1 \%$ for veterans of recent conflicts. $^{2}$ PTSD and subthreshold symptomatology often become chronic and are associated with decrements in physical, financial and psychological well-being. ${ }^{3-6}$ While there are several psychological treatments for PTSD with proven efficacy, these treatments do not help all people who suffer from PTSD. Dropout and non-response rates for traditional PTSD treatments are high ${ }^{7}$ and proven treatments may have lower efficacy in veteran samples. ${ }^{9}$ An additional barrier to receipt of treatment is that individuals with PTSD may not seek conventional 'talk therapy' due to stigma associated with engaging with a mental health system. ${ }^{10}$ 
Thus, it is critical to investigate other therapies that appeal to veterans with PTSD.

Complementary and integrative treatments, such as mindfulness meditation and acupuncture, have received increasing attention in clinical settings as alternative or adjunctive treatments for PTSD. Recent studies indicate that over $30 \%$ of individuals diagnosed with PTSD use complementary and integrative treatments for mental health issues ${ }^{11}$ and almost all Veterans Health Administration specialised PTSD programmes offer some integrative therapies. ${ }^{12}$ The Institute of Medicine (IOM) recently called for more research on complementary and integrative approaches to the treatment of PTSD ${ }^{13}$ and randomised controlled trials are accumulating to support the use of integrative therapies. New evidence indicates that yoga ${ }^{14}$ and mindfulness interventions ${ }^{15}$ are efficacious treatments for PTSD. Tai Chi as a treatment to address PTSD symptoms has not yet been systematically evaluated, although preliminary case studies suggest it may be efficacious. ${ }^{16}$

Tai Chi is an ancient Chinese form of exercise involving slow, gentle movements and diaphragmatic breathing. Derived from the martial arts, Tai Chi is also a mind-body practice that incorporates mindfulness, breathing, active relaxation and deliberate movements. The characteristic slow movement allows individuals with chronic conditions and varying levels of fitness to participate. ${ }^{17}$ In addition to physical improvements in flexibility, strength and pain management, ${ }^{17}$ there is evidence that Tai Chi improves sleep ${ }^{18}$ and reduces depression and anger. ${ }^{19} 20$

Tai Chi may impact PTSD symptoms via different mechanisms of action. First, as with other mind-body practices (yoga, mindfulness meditation), Tai Chi encourages a focus on the present moment and awareness of body positions and sensations. The slow, deliberate movement with simultaneous mindful attention to one's breathing and body may promote relaxation, ${ }^{21}$ and enhance regulation of physiological arousal..$^{22}$ Thus, Tai Chi may impact the PTSD hyperarousal symptoms that have been identified as prominent in the development and maintenance of the disorder. ${ }^{23}{ }^{24}$ Second, the proven efficacy of Tai Chi to address symptoms of pain and depression and to improve function and psychological well-being ${ }^{17} 2025$ may slow the progression of disease and disability associated with PTSD. This is particularly plausible since chronic pain, depression and health ailments commonly accompany PTSD ${ }^{26-28}$ and, left untreated, are associated with increased nonresponse to PTSD treatment. ${ }^{29}$ Finally, for veterans with PTSD who may have traumatic associations with combat stances and postures, warrior stances combined with calm breathing and the gentle motion of Tai Chi may provide an opportunity for positive associations with warrior identities to emerge and develop.

Although Tai Chi's positive effects on general wellbeing have been well documented, ${ }^{20}{ }^{25}$ Tai Chi has not yet been examined as an intervention for PTSD or subthreshold symptoms of PTSD. The current investigation represents a preliminary step towards a systematic evaluation of Tai Chi as a treatment for PTSD. The foursession introduction to Tai Chi used in the current study was a brief 'taste' of Tai Chi and substantially shorter than a typical Tai Chi programme of 24 or more sessions. The aims of this study were: (1) to introduce Tai Chi to veterans with symptoms of PTSD and examine subjective reactions to this novel intervention, (2) to examine recruitment, participant characteristics, adherence, adverse events and satisfaction associated with a brief Tai Chi intervention in order to provide information to support a future clinical trial.

\section{METHODS}

\section{Procedures}

This study was conducted at a large Veterans Affairs (VA) Healthcare System in the Northeastern USA in collaboration with a principal investigator at an affiliated university. We used flyers, brochures, referrals from clinicians, and a participant recruitment database for recruitment. Participants were compensated $\$ 40$ for each assessment, $\$ 20$ for the focus group, $\$ 20$ for the individual interview, and $\$ 10$ for travel for each Tai Chi session attended. Study staff completed the informed consent process and all participants signed consent forms. At the initial visit, participants completed self-report measures.

\section{Participants}

Seventeen veterans with post-traumatic stress symptoms enrolled in the Tai Chi programme. Two cohorts consisting of up to nine veterans participated in a four-session Tai Chi protocol designed to provide a brief introduction to Tai Chi exercise. After the final Tai Chi session, participants completed a satisfaction questionnaire and provided feedback to investigators via a focus group and individual interviews using open-ended questions. As cohorts were run sequentially, we used feedback obtained from the first cohort to modify the study protocol for the second cohort.

Individuals with subthreshold PTSD symptomatology frequently experience significant impairment in functioning. ${ }^{6}$ Thus, we included both veterans with subthreshold symptomatology as well as veterans with diagnosed PTSD in order to represent the full range of veterans with postdeployment trauma-related distress. Inclusion criteria were (1) at least 18 years of age, (2) a PTSD diagnosis in the electronic medical record or endorsement of at least one of the Diagnostic and Statistical Manual of Mental Disorders, Fifth Edition (DSM-5) re-experiencing symptoms of PTSD during the telephone screening, (3) willingness to complete five visits over the course of the study, (4) no plans to relocate while enrolled in the study, (5) agreement not to initiate enrolment in other formalised exercise programmes until completion of the month long study, (6) no current medical conditions that limit ability to 
participate safely in moderate exercise as measured by the Physical Activity Readiness Questionnaire (PAR-Q) ${ }^{30}$ during the telephone screening, (7) no contraindications noted by the participant's primary care provider (or another member of the medical team, eg, cardiologist) and (8) not currently pregnant, if female.

Exclusion criteria were (1) evidence of active substance dependence as noted in the electronic medical record, (2) a current diagnosis of mania, hypomania, unstable bipolar disorder, psychotic disorder, active suicidality or a history of psychosis, as noted in electronic medical record, (3) recent (past 3 months) hospitalisation for mental health or substance use issues and (4) current participation in another longitudinal intervention study addressing post-traumatic stress symptoms, traumatic brain injury or other mental health disorders.

\section{Description of sample}

Demographic information, height and weight were collected at the initial visit (see table 1). Three psychometric self-report measures were administered to provide descriptive information about participants: the PTSD Checklist for DSM-5 (PCL-5), ${ }^{31-33}$ the Brief Pain InventoryShort Form (BPI) ${ }^{34} 35$ and the Beck Depression Inventory II (BDI-II) ${ }^{36}{ }^{37}$ To assess current use of medications, participants were provided with a list of psychotropic and pain medications and asked to check off medications they were currently taking and provide the dose. For veterans who receive medications through the VA, the medications and doses were verified via electronic medical record. Medical records were also examined to determine if participants were engaged in group or individual psychological treatment during or in the month before or after the Tai Chi programme.

\section{Tai Chi sessions}

Tai Chi sessions occurred once a week for 4 weeks, with the first session lasting up to $90 \mathrm{~min}$ and the remaining three sessions each lasting $60 \mathrm{~min}$. Two trained Tai Chi instructors, each with over 20 years of experience teaching Tai Chi mind-body programmes, conducted all Tai Chi sessions. One instructor led all four sessions for the first cohort and the other instructor led all four sessions for the second cohort.

The Tai Chi instructors followed a standardised Tai Chi protocol and at each session provided participants with printed materials on Tai Chi principles and practice techniques. In the first class, the instructor explained the exercise theory and procedures of Tai Chi and reviewed printed materials with participants. Every session included the following components: (1) warm-up, self-massage and a review of Tai Chi principles; (2) Tai Chi movement; (3) breathing techniques; and (4) relaxation. Each component of the programme was derived from classical Yang style Tai Chi. The instructors also encouraged participants to practice Tai Chi for at least $30 \mathrm{~min}$ a day at home. Participants were asked to complete daily practice logs after home practice that indicated days they practiced, minutes of practice and which exercises were practiced.

\section{Qualitative focus groups and interviews}

After the final session of Tai Chi, a brief focus group was conducted with each cohort of participants. Each focus group was led by two doctoral-level clinicians and was audiotaped. Focus group leaders asked open-ended questions about the following topics: (1) satisfaction with the Tai Chi intervention and instructor, (2) comfort with participating in a research study, (3) group dynamics, (4) class size, (5) impact of Tai Chi on post-traumatic stress symptoms and (6) future use of Tai Chi. In addition, study staff interviewed participants individually after the focus group to provide an opportunity for participants to report additional feedback in privacy.

\section{Satisfaction}

Following the final Tai Chi session, participants were asked to respond to eight written satisfaction questions on a four-point Likert-type scale. These questions were informed by the Client Satisfaction Questionnaire $(\mathrm{CSQ})^{38}$ and modified substantially to assess satisfaction specific to the Tai Chi intervention.

\section{Data analysis plan}

Descriptive and summary statistics were used to examine recruitment, participant characteristics, adherence and satisfaction.

Qualitative analysis was conducted using an iterative coding process. Qualitative data (from focus groups and interviews) were transcribed and then analysed by a committee of reviewers using a general inductive approach. ${ }^{39}$ This committee consisted of three research psychologists, one master's-level research trainee and two bachelor's-level research assistants. Raters independently coded several transcripts to identify themes. Next, the committee met to review identified themes, to discuss discrepancies and to refine the coding rubric. Raters coded the remaining transcripts and continued to meet until consensus was reached on the identified themes and on the distribution of participant quotes under appropriate themes. The committee then chose participant quotes that best represented the identified themes for inclusion in the current manuscript.

\section{RESULTS}

\section{Recruitment}

Figure 1 presents a flow diagram of study participation.

Twenty-seven veterans completed telephone screening. Of these, 8 veterans were ineligible $(29.6 \%)$, 2 were not interested $(7.4 \%)$ and $17(63.0 \%)$ enrolled in the study. It is notable that recruitment for the first cohort in May 2014 was so successful that we received more referrals and calls from interested veterans than we could accommodate in the first cohort. Thus, no additional recruitment efforts were needed to fill the second cohort in June 2014. 
Table 1 Participant characteristics at baseline $(\mathrm{N}=17)$

\begin{tabular}{|c|c|c|c|}
\hline Variable & n (\%) & $M(S D)$ & Range \\
\hline Age & & $51.12(9.78)$ & $32-67$ \\
\hline \multicolumn{4}{|l|}{ Gender } \\
\hline Male & $11(64.7)$ & & \\
\hline Female & $6(35.3)$ & & \\
\hline \multicolumn{4}{|l|}{ Ethnicity } \\
\hline Hispanic or Latino & $1(5.9)$ & & \\
\hline Non-Hispanic or Latino & $15(88.2)$ & & \\
\hline Unknown (not reported) & $1(5.9)$ & & \\
\hline \multicolumn{4}{|l|}{ Race } \\
\hline Black or African-American & $4(23.5)$ & & \\
\hline White & $10(58.8)$ & & \\
\hline More than one race & $3(17.6)$ & & \\
\hline \multicolumn{4}{|l|}{ Service era* } \\
\hline Vietnam & $2(11.8)$ & & \\
\hline Post-Vietnam & $7(41.1)$ & & \\
\hline Gulf war & $5(29.4)$ & & \\
\hline OEF/OIF/OND & $6(35.3)$ & & \\
\hline \multicolumn{4}{|l|}{ Marital/partner status } \\
\hline Married/living with partner & $8(47.1)$ & & \\
\hline Divorced/separated & $3(17.6)$ & & \\
\hline Widowed & $1(5.9)$ & & \\
\hline Never married & $5(29.4)$ & & \\
\hline \multicolumn{4}{|l|}{ Education } \\
\hline High school Diploma/GED & $1(5.9)$ & & \\
\hline Some college/trade or technical training & $11(64.7)$ & & \\
\hline College degree ( 4 years) & $3(17.6)$ & & \\
\hline Attended/completed graduate school & $2(11.8)$ & & \\
\hline \multicolumn{4}{|l|}{ Employment } \\
\hline Working full time & $5(29.4)$ & & \\
\hline Unemployed and looking for work & $2(11.8)$ & & \\
\hline Disabled and not working & $9(52.9)$ & & \\
\hline Retired and not working & $1(5.9)$ & & \\
\hline BMI & & $31.79(4.80)$ & $26.6-41.6$ \\
\hline PCL-5 & & $44.56(17.78)$ & $11-74$ \\
\hline Suggestive of PTSD diagnosis† & $13(76.5)$ & & \\
\hline Subthreshold symptoms & $4(23.5)$ & & \\
\hline \multicolumn{4}{|l|}{ Brief Pain Inventory } \\
\hline Currently experiencing pain & $11(64.7)$ & & \\
\hline Pain severity score $\ddagger$ & & $4.67(2.79)$ & $0-9.25$ \\
\hline Pain interference score§ & & 4.75 (3.33) & $0-10$ \\
\hline Beck Depression Inventory & & $24.56(11.87)$ & $3-44$ \\
\hline Normative & $3(17.6)$ & & \\
\hline Mild/borderline depression & $3(17.6)$ & & \\
\hline Moderate depression & $4(23.5)$ & & \\
\hline Severe or extreme depression & $7(41.1)$ & & \\
\hline Psychological treatment (individual and/or group) & $11(64.7 \%)$ & & \\
\hline Pain medications* & $15(88.2)$ & & \\
\hline Analgesics & $7(41.2)$ & & \\
\hline Daily NSAIDs other than aspirin & $7(41.2)$ & & \\
\hline Narcotics (occasionally to daily) & $8(47.1)$ & & \\
\hline Anticonvulsants for nerve pain & $5(29.4)$ & & \\
\hline Muscle relaxants & $1(5.9)$ & & \\
\hline Psychotropic medications ${ }^{*}$ & $13(76.5)$ & & \\
\hline Antidepressants/anxiety/sleep & $11(64.7)$ & & \\
\hline Antipsychotics & $2(11.8)$ & & \\
\hline Mood stabilisers & $1(5.9)$ & & \\
\hline Benzodiazepines & $5(29.4)$ & & \\
\hline
\end{tabular}

Some percentages do not add up to $100 \%$ due to rounding.

*Service era, pain medications and psychotropic medications are not mutually exclusive.

†A total score of 33 or more on the PCL-5.

$\ddagger 0=$ No pain; $10=$ pain as bad as you can imagine.

$\S 0=$ Does not interfere; $10=$ completely interferes.

BMI, body mass index; GED, General Education Diploma; NSAID, non-steroidal anti-inflammatory drug; OEF, Operation Enduring Freedom; OIF, Operation Iraqi Freedom; OND, Operation New Dawn; PCL, PTSD Checklist; PTSD, post-traumatic stress disorder. 
Figure 1 Flow chart of participant completion rates.

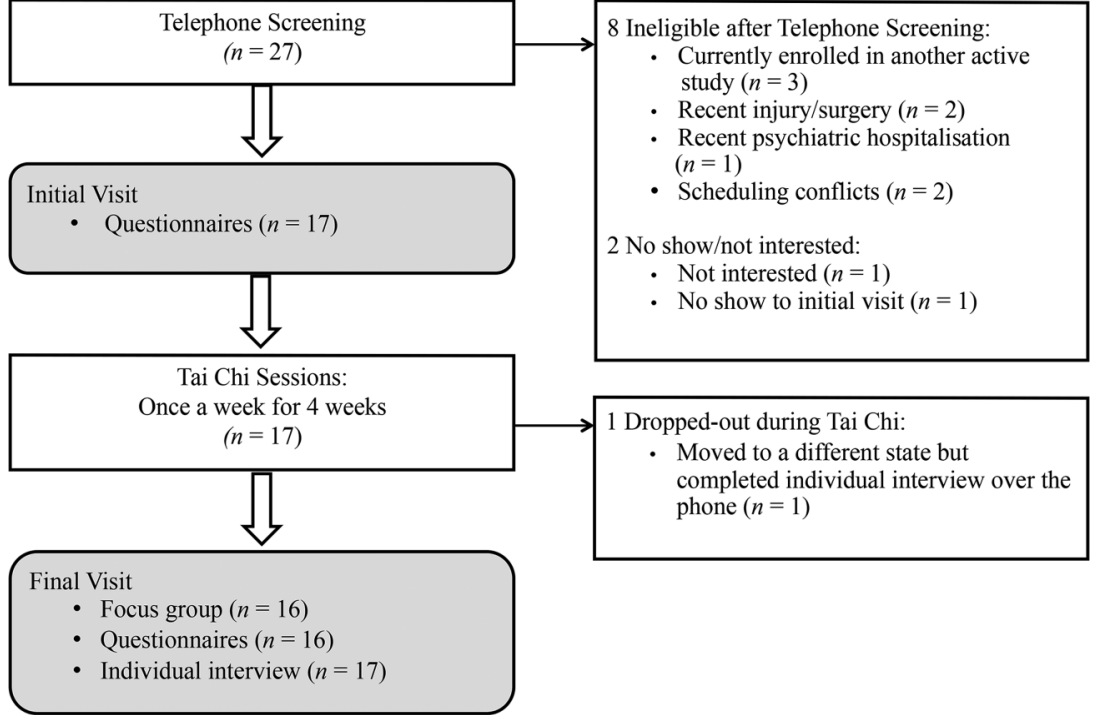

\section{Participant characteristics}

Seventeen veterans (11 males, 6 females; mean age $=51.12$, range $=32-67$ ) with post-traumatic stress symptoms participated in the study (see table 1 ).

All participants endorsed at least one of the hallmark re-experiencing symptoms of PTSD, with $64.7 \%$ endorsing symptoms suggestive of a PTSD diagnosis. Notably, these veterans had an average body mass index (BMI) in the obese range (31.8) and all participants were in the overweight or obese range. Approximately two-thirds of the participants reported they were experiencing pain $(64.7 \%)$ and the same number reported moderate-to-severe symptoms of depression (64.7\%). Approximately two-thirds $(64.7 \%)$ were engaged in individual and/or group psychological treatment. Most

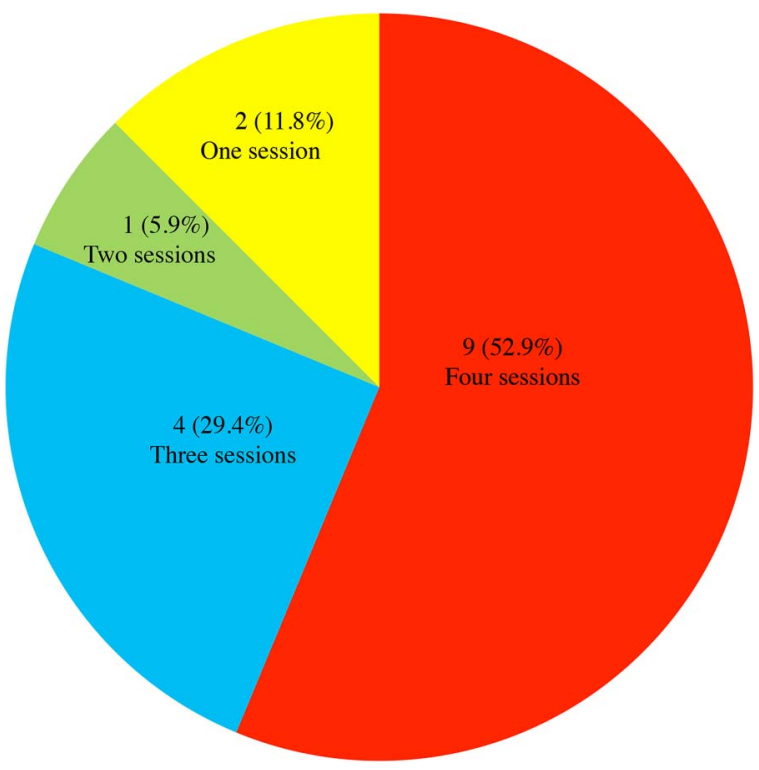

Figure 2 Participant attendance. participants $(88.2 \%)$ were prescribed medications for pain and almost half of the total sample was prescribed narcotics $(47.1 \%)$. The majority of participants were taking at least one psychotropic medication (76.5\%) and almost a third of all participants $(29.4 \%)$ were prescribed benzodiazepines.

\section{Adherence and retention}

Participants attended the majority of the Tai Chi sessions (14 of 17 or $82.3 \%$ attended three or four sessions; see figure 2).

One participant unexpectedly relocated to a different state and was able to attend only one Tai Chi session, but provided feedback in a telephone interview at the completion of the study. Another participant only attended the final session due to difficulties with transportation and scheduling. Attendance at sessions 1-4 was 14 (82.3\%), 15 (88.2\%), $10(58.8 \%)$ and 16 (94.1\%), respectively. All 17 participants provided feedback via individual interview. Sixteen participants (94.1\%) completed the post-treatment questionnaires and focus group. Rates of practice log completion were low for the first cohort and this prompted a change in the protocol. In the second cohort, the instructor and study staff put more emphasis on the practice logs during and following each session, resulting in higher completion rates. In the first cohort, $33.3 \%$ of participants (3/9) completed at least two (of three possible) practice logs whereas $75.0 \%$ of the participants in the second cohort (6/8) completed at least two practice logs.

\section{Adverse events}

No adverse events were reported for this study. In response to the satisfaction question about whether the Tai Chi programme helped participants to deal more effectively with their problems, none $(0 \%)$ of the participants reported that Tai Chi made things worse. 


\section{Qualitative findings}

Table 2 summarises the identified themes and subcategories as well as the participant quotes that best represented the themes.

General satisfaction/benefits of Tai Chi: Participants appeared to be very satisfied with the introduction to Tai Chi. The majority of participants commented on the relaxing nature of Tai Chi and identified other benefits as well. One woman who had experienced military sexual trauma reported that she felt surprisingly at ease in the class.

Perception of Tai Chi: Participants had a favourable impression of Tai Chi and found it comfortable. A few identified the martial arts component of Tai Chi as particularly appealing.

Participant engagement: Generally, participants were engaged by the introductory Tai Chi programme and were able to keep their attention focused on the exercises.

Benefits for post-traumatic stress symptoms, depression and pain: Participants found Tai Chi to be helpful with managing distressing symptoms. One veteran reported having decreased intrusive visions or flashbacks over the course of the study. Other participants commented on how Tai Chi was a helpful stress reduction technique. Two veterans indicated that doing the Tai Chi exercise was physically challenging, but that it was manageable and ultimately positive (eg, loosens muscles, good workout).

Tai Chi instructors: Overall, participants praised the instructors and appreciated their positive energy, supportive comments and the consistent structure of the classes. Some noted that they liked the individual attention and the modification of movements based on individual need.

Tai Chi home practice: Some participants said they practiced Tai Chi at home and found it helpful. One participant involved a significant other in the home practice in order to share the practice with her. Another veteran reported practising Tai Chi even more than the instructors suggested.

Suggestions for improvement: Participants made suggestions on how the Tai Chi programme could be enhanced. First, some participants reported that it was difficult to recreate the Tai Chi movements by looking at the pictures provided in the home practice handouts. Several participants also stated that they would have liked a longer programme and most of the participants indicated that they wanted more space in the Tai Chi group room. Others suggested that, in addition to the instructor, it would be helpful to have a research staff member in the room to handle participant concerns in order to minimise class interruptions.

\section{Satisfaction questionnaire findings}

General satisfaction with the brief introduction to Tai Chi intervention was very high; $93.8 \%(15 / 16)$ of veterans reported that they were very or mostly satisfied with the four-session programme, that they were satisfied with 'the amount of help they received', and that they would rate the 'quality of the programme' as excellent or good. In addition, $87.5 \%(14 / 16)$ endorsed that they got 'the kind of experience they wanted' in the Tai Chi programme and $81.3 \%(13 / 16)$ reported that most or almost all 'of their needs were met' with the Tai Chi programme. Eleven of the participants $(68.8 \%)$ endorsed that the four-session introduction to Tai Chi helped them to 'deal more effectively with their problems'-an unanticipated finding given the brevity of the programme. All participants (100\%) endorsed that they would 'recommend the Tai Chi programme to a friend' and that they would return for more Tai Chi if such a programme were offered again.

\section{DISCUSSION}

Veterans reported being highly satisfied with the Tai Chi intervention, both on written satisfaction questionnaires and when interviewed alone and as a group. Veterans indicated that the programme helped with several symptoms, such as intrusive symptoms, concentration difficulties and physiological arousal. In the open-ended interviews, several participants spoke of feeling unusually focused during the Tai Chi sessions and feeling calmed during and after sessions. Interestingly, participants commented that Tai Chi, a martial art, resonated with their 'warrior spirit' in a positive way. The strong endorsement of the Tai Chi programme was highlighted by the fact that all participants reported that they would recommend the Tai Chi programme to a friend and that they would be interested in returning for more.

Results from this study indicate that recruiting veterans to participate and retaining them in a four-session introduction to Tai Chi intervention for post-traumatic stress symptoms is feasible. Recruitment was remarkably successful: almost $90 \%(17 / 19)$ of those eligible following the telephone screen enrolled in the programme. Attendance in this study was good, as most participants $(82.3 \%)$ attended at least three of the four Tai Chi sessions offered. Retention was excellent, as all participants completed at least some portion of the final feedback session. These rates of recruitment, attendance and retention compare favourably to studies of treatments for veterans with PTSD symptoms, where dropout rates are frequently high and range up to $50 \% .{ }^{78}$ In comparison to other studies of complementary and integrative health treatments, our rates are good. For example, a recent large-scale study of a nine-session mindfulness intervention for veterans with PTSD reported that $81 \%$ completed treatment and participants attended $77.3 \%$ of the sessions. ${ }^{15}$ Although compliance with home practice and practice logs in the first cohort was low, it was substantially higher in the second cohort following alterations to the protocol that placed greater emphasis on home practice. This illustrates that adapting the 
Table 2 Qualitative findings

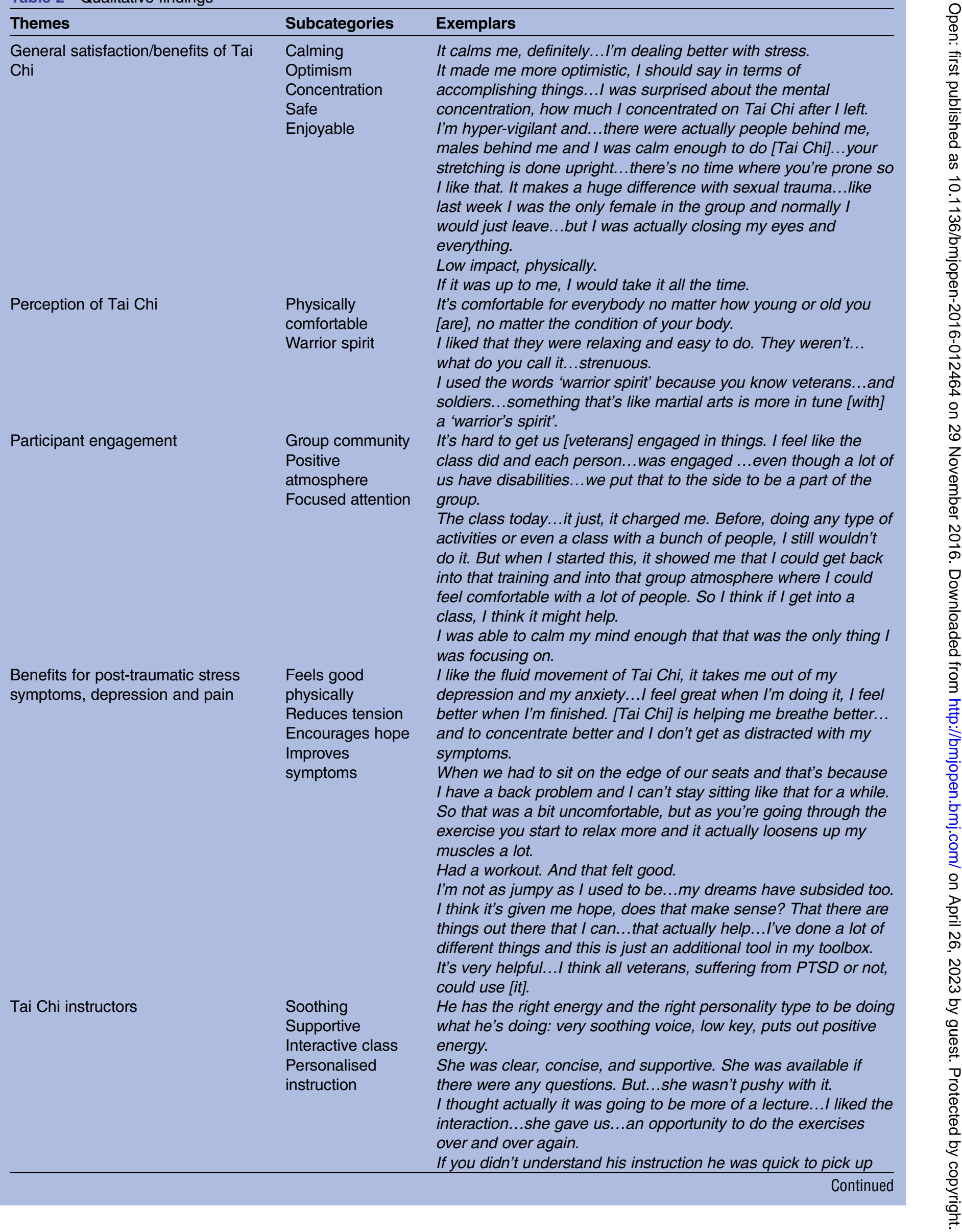


Table 2 Continued

\begin{tabular}{|c|c|c|}
\hline Themes & Subcategories & Exemplars \\
\hline & & $\begin{array}{l}\text { and [would] come over and correct you so it was fine. Or you } \\
\text { could ask him a question and he'd help you. } \\
\text { She modified a couple movements for me and...she gave me } \\
\text { attention [while] watching me perform it. }\end{array}$ \\
\hline \multirow[t]{2}{*}{ Tai Chi home practice } & Relaxing & When I do it at home, it's relaxing. It helps. \\
\hline & Positive routine & $\begin{array}{l}\text { I was doing half an hour in the morning, half an hour in the } \\
\text { afternoon, half an hour in the evenings...I was doing an hour and } \\
\text { a half a day. } \\
\text { Yes, my significant other, she does it with me. }\end{array}$ \\
\hline \multirow[t]{3}{*}{ Suggestions for improvement } & $\begin{array}{l}\text { Video for home } \\
\text { practice }\end{array}$ & $\begin{array}{l}\text { It'd be nice if they could give you a video to take home, that you } \\
\text { could play and while you're doing it, at your own place. }\end{array}$ \\
\hline & $\begin{array}{l}\text { Larger space } \\
\text { More sessions }\end{array}$ & $\begin{array}{l}\text { Of course a bigger room.I found that was really short...just the } \\
\text { four weeks of [Tai Chi]. }\end{array}$ \\
\hline & Staff in room & $\begin{array}{l}\text { Maybe, yeah if there was somebody else there, a staff member } \\
\text { there and like not as knowledgeable about Tai Chi but knowing } \\
\text { what he's trying to do and say if you're uncomfortable or if you're } \\
\text { having problems, whatever, address it to me and let the } \\
\text { instructor continue on with the class. }\end{array}$ \\
\hline
\end{tabular}

study protocol successfully encouraged participant engagement in the programme. Additional adaptations, such as having a study staff member in the room during Tai Chi sessions to address concerns could further enhance engagement for individuals with post-traumatic stress symptoms.

Despite the fact that all of the participants in this study had BMIs that were in the overweight or obese range and the majority had symptoms of pain and/or depression and were taking prescribed medications to address these problems, there were no adverse events reported during the course of the study. This may be attributed to the slow, gentle movements of Tai Chi and to the instructors' skill in tailoring the intervention to the ability of each individual. Of note, some participants reported a slight increase in pain during or immediately following the exercises, and one described this as a tolerable pain that 'felt good'. Participants also commented that Tai Chi is a physical activity that is comfortable for a broad range of people, regardless of physical condition. This further supports the feasibility and safety of this type of programme for veterans with post-traumatic stress symptoms who have high rates of obesity, ${ }^{40}{ }^{41}$ chronic pain ${ }^{27}$ and other physical health problems. ${ }^{28}$

There were several important limitations to this study. First, the sample size was small, the study did not use a diagnostic measure, and some of the participants reported mild symptoms of PTSD, so findings may not apply to a broader population of veterans with diagnosed PTSD. Additionally, there may have been a selection bias. Since participants understood they were volunteering to take part in a Tai Chi study, they were likely more enthusiastic about participating and offering positive feedback about this unconventional intervention than others within the larger veteran population. Also, the monetary compensation provided to participants for transportation, time and inconvenience may have acted as an incentive for participation and potentially enhanced recruitment, attendance and retention rates. However, recent research with veterans indicates that these remuneration rates are consistent with current recommendations. ${ }^{42}$ Finally, the four-session introduction to Tai Chi was substantially shorter than a typical Tai Chi programme. It may be more difficult to enrol and retain participants in a longer or more intensive intervention. Alternatively, a lengthier intervention may allow participants to experience increasing physical and psychological benefits from Tai Chi, leading to continued attendance.

Despite these limitations, this study provides evidence for the feasibility of enrolling and engaging veterans with symptoms of PTSD in a Tai Chi exercise programme. Findings also indicate that Tai Chi is a safe physical activity and appropriate for individuals with varying physical capabilities. Veterans were very satisfied with their Tai Chi experience and they indicated both willingness and a preference for additional sessions. Given these positive findings, additional research is needed to empirically evaluate Tai Chi as a treatment for symptoms of PTSD. Future rigorous studies testing the Tai Chi programme should use larger trials with control groups and random assignment. This investigation identified potential mechanisms through which Tai Chi influences PTSD symptoms (eg, reducing physiological arousal, improving comorbid conditions, increasing positive associations with warrior identity) to be examined in future research. This study highlights the need for future investigation of Tai Chi as a novel intervention to address symptoms of PTSD. 
Author affiliations

${ }^{1}$ National Center for PTSD, VA Boston Healthcare System and Boston

University School of Medicine, Boston, Massachusetts, USA

${ }^{2}$ VA Boston Healthcare System and Boston University School of Medicine,

Boston, Massachusetts, USA

${ }^{3}$ National Center for PTSD and VA Boston Healthcare System, Boston,

Massachusetts, USA

${ }^{4}$ Division of Rheumatology, Center for Integrative Medicine, Tufts Medical

Center, Tufts University School of Medicine, Boston, Massachusetts, USA

Acknowledgements The authors wish to thank the veterans who participated and Tai Chi instructors Brian Muccio and Dorothy Li for their expertise, time and effort

\section{Collaborators Brian Muccio Dorothy Li.}

Contributors CW was the principal investigator (PI) and BLN and DLM were co-PIs at the VA Boston Healthcare System (VABHS) site. APK, CPP and AML made substantial contributions to the design and management of the study. CPP was primarily responsible for recruitment and data collection. All authors made substantial contributions to drafts of the manuscript and all have read and approved the final manuscript.

Funding This study was supported by the National Center for Complementary and Integrative Health (NCCIH; R01AT006367-01A1 and K24AT007323) and funds from the National Center for PTSD.

Disclaimer The investigators are solely responsible for the contents of the manuscript and they do not represent official views of the $\mathrm{NCCIH}$, the US Department of Veterans Affairs or the US Government.

Competing interests None declared.

Ethics approval The Institutional Review Board (IRB) committees at the VA Boston Healthcare System and Tufts Medical Center approved the participation of human participants.

Provenance and peer review Not commissioned; externally peer reviewed.

Data sharing statement Summary data may be available from the first author on request.

Open Access This is an Open Access article distributed in accordance with the Creative Commons Attribution Non Commercial (CC BY-NC 4.0) license, which permits others to distribute, remix, adapt, build upon this work noncommercially, and license their derivative works on different terms, provided the original work is properly cited and the use is non-commercial. See: http:// creativecommons.org/licenses/by-nc/4.0/

\section{REFERENCES}

1. Kessler RC, Berglund $\mathrm{P}$, Demler $\mathrm{O}$, et al. Lifetime prevalence and age-of-onset distributions of DSM-IV disorders in the National Comorbidity Survey Replication. Arch Gen Psychiatry 2005;62:593-602

2. Fulton JJ, Calhoun PS, Wagner HR, et al. The prevalence of posttraumatic stress disorder in Operation Enduring Freedom/ Operation Iraqi Freedom (OEF/OIF) Veterans: a meta-analysis. $J$ Anxiety Disord 2015;31:98-107.

3. Engelhard IM, van den Hout MA, Weerts $\mathrm{J}$, et al. A prospective study of the relation between posttraumatic stress and physical health symptoms. Int J Clin Health Psychol 2009;9:365-72.

4. Sanderson K, Andrews G. Common mental disorders in the workforce. Recent findings from descriptive and social epidemiology Can J Psychiatr 2006;51:63-75.

5. Sareen J, Cox BJ, Stein MB, et al. Physical and mental comorbidity, disability, and suicidal behavior associated with posttraumatic stress disorder in a large community sample. Psychosom Med 2007:69:242-8.

6. Mota NP, Tsai J, Sareen J, et al. High burden of subthreshold DSM-5 post-traumatic stress disorder in U.S. military Veterans. World Psychiatry 2016;15:185-6.

7. Imel ZE, Laska K, Jakupcak M, et al. Meta-analysis of dropout in treatments for posttraumatic stress disorder. J Consult Clin Psychol 2013:81:394-404

8. Schottenbauer MA, Glass CR, Arnkoff DB, et al. Nonresponse and dropout rates in outcome studies on PTSD: review and methodological considerations. Psychiatry 2008;71:134-68.
9. Steenkamp MM, Litz BT. Psychotherapy for military-related posttraumatic stress disorder: review of the evidence. Clin Psychol Rev 2013;33:45-53.

10. Hoge CW, Castro CA, Messer SC, et al. Combat duty in Iraq and Afghanistan, mental health problems, and barriers to care. N Engl J Med 2004;351:13-22.

11. Libby DJ, Pilver CE, Desai R. Complementary and alternative medicine use among individuals with posttraumatic stress disorder Psychol Trauma Theory Res Pract Policy 2013;5:277-85.

12. Libby DJ, Pilver CE, Desai R. Complementary and alternative medicine in VA specialized PTSD treatment programs. Psychiatr Serv 2012;63:1134-6.

13. IOM (Institute of Medicine). Treatment for posttraumatic stress disorder in military and veteran populations: initial assessment. Washington DC: The National Academies Press, 2012.

14. van der Kolk B, Stone $\mathrm{L}$, West $\mathrm{J}$, et al. Yoga as an adjunctive treatment for posttraumatic stress disorder: a randomized controlled trial. J Clin Psychiatr 2014;75:559-65.

15. Polusny MA, Erbes CR, Thuras P, et al. Mindfulness-based stress reduction for posttraumatic stress disorder among veterans: a randomized clinical trial. JAMA 2015;314:456-65.

16. Grodin MA, Piwowarczyk L, Fulker D, et al. Treating survivors of torture and refugee trauma: a preliminary case series using qigong and t'ai chi. J Altern Complemt Med 2008;14:801-6.

17. Wang C, Collet JP, Lau J. The effect of Tai Chi on health outcomes in patients with chronic conditions: a systematic review. Arch Intern Med 2004;164:493-501.

18. Raman G, Zhang Y, Minichiello VJ, et al. Tai Chi improves sleep quality in healthy adults and patients with chronic conditions: a systematic review and meta-analysis. J Sleep Disord Ther 2013;2:141. http://omicsgroup.org/journals/tai-chi-improves-sleepquality-in-healthy-adults-and-patients-with-chronic-conditions-asystematic-review-and-metaanalysis-2167-0277-2-141.pdf (accessed 21 Jan 2016)

19. Jin P. Changes in heart rate, noradrenaline, cortisol and mood during Tai Chi. J Psychosom Res 1989:33:197-206.

20. Wang C, Bannuru R, Ramel J, et al. Tai Chi on psychological well-being: systematic review and meta-analysis. BMC Complemt Altern Med 2010;10:1-16.

21. Kim SH, Schneider SM, Kravitz L, et al. Mind-body practices for posttraumatic stress disorder. J Invest Med 2013;61: 827-34.

22. Brown KW, Ryan RM. The benefits of being present: mindfulness and its role in psychological well-being. $J$ Pers Soc Psychol 2003;84:822-48.

23. Schell TL, Marshall GN, Jaycox LH. All symptoms are not created equal: the prominent role of hyperarousal in the natural course of posttraumatic psychological distress. J Abnorm Psychol 2004;113:189-97.

24. Doron-LaMarca S, Niles BL, King DW, et al. Temporal associations among chronic PTSD symptoms in U.S. combat veterans. J Trauma Stress 2015;28:410-17.

25. Wang $\mathrm{C}$, Schmid $\mathrm{CH}$, Rones $\mathrm{R}$, et al. A randomized trial of tai chi for fibromyalgia. N Engl J Med 2010;363:743-54.

26. Campbell DG, Felker BL, Liu CF, et al. Prevalence of depressionPTSD comorbidity: implications for clinical practice guidelines and primary care-based interventions. J Gen Intern Med 2007;22: 711-18.

27. Otis JD, Gregor K, Hardway C, et al. An examination of the co-morbidity between chronic pain and posttraumatic stress disorder on US Veterans. Psychol Serv 2010;7:126-35.

28. Pacella ML, Hruska B, Delahanty DL. The physical health consequences of PTSD and PTSD symptoms: a meta-analytic review. J Anxiety Disord 2013;27:33-46.

29. Markowitz JC, Petkova E, Neria $Y$, et al. Is exposure necessary? A randomized clinical trial of interpersonal psychotherapy for PTSD. Am J Psychiatr 2015;172:430-40.

30. Chisholm DM, Collis ML, Kulak LL, et al. Physical activity readiness. Br Columbia Med J 1975;17:375-8.

31. Weathers FW, Litz BT, Herman DS, et al. The PTSD checklist (PCL-5): reliability, validity, and diagnostic utility. Paper presented at the meeting of the International Society of Traumatic Stress Studies. San Antonio, Texas, 1993.

32. Keen SM, Kutter CJ, Niles BL, et al. Psychometric properties of PTSD Checklist in sample of male veterans. J Rehabil Res Dev 2008;45:465-74.

33. Weathers F, Litz B, Keane T, et al. PTSD Checklist for DSM-5 (PCL-5). 2014. http://www.ptsd.va.gov/professional/assessment/ adult-sr/ptsd-checklist.asp (accessed 21 Jan 2016).

34. Cleeland C. The brief pain inventory user guide. Houston, TX: The University of Texas MD Anderson Cancer Center, 2009. 
35. Cleeland CS, Ryan KM. Pain assessment: global use of the brief pain inventory. Ann Acad Med Singapore 1994;23: 129-38.

36. Beck AT, Steer RA, Brown GK. Beck Depression Inventory-II (BDI-II). Manual for Beck Depression Inventory-II. 1993.

37. Storch EA, Roberti JW, Roth DA. Factor structure, concurrent validity, and internal consistency of the beck depression inventorysecond edition in a sample of college students. Depress Anxiety 2004;19:187-9.

38. Larsen DL, Attkisson CC, Hargreaves WA, et al. Assessment of client/patient satisfaction: development of a general scale. Eval Program Plann 1979;2: 197-207.

39. Thomas DR. A general inductive approach for analyzing qualitative evaluation data. Am J Eval 2006;27:237-46.
40. Pagoto SL, Schneider KL, Bodenlos JS, et al. Association of post-traumatic stress disorder and obesity in a nationally representative sample. Obesity (Silver Spring) 2012;20: 200-5.

41. Farr OM, Ko BJ, Joung KE, et al. Posttraumatic stress disorder, alone or additively with early life adversity, is associated with obesity and cardiometabolic risk. Nutr Metab Cardiovas 2015;25:479-88.

42. Office of Research and Development, U.S. Department of Veteran Affairs. How to engage Iraq, Afghanistan Veterans in health research: lessons from focus groups. U.S. Department of Veteran Affairs Office of Research and Development, Research Currents, 2016. http://www.research.va.gov/currents/0416-5.cfm (accessed 3 Aug 2016). 